\title{
Double motion of upper eyelids in Graves' orbitopathy: an additional sign for detection of thyroid dysfunction or positive thyroid autoantibodies
}

\author{
This article was published in the following Dove Press journal: \\ Clinical Ophthalmology \\ 3 March 2011 \\ Number of times this article has been viewed
}

\section{Hirohiko Kakizaki' \\ Yasuhiro Takahashi' \\ Masayoshi Iwaki' \\ Akihiro Ichinose ${ }^{2}$ \\ Dinesh Selva ${ }^{3}$ \\ Igal Leibovitch ${ }^{4}$}

'Department of Ophthalmology, Aichi Medical University, Nagakute, Aichi, Japan; ${ }^{2}$ Department of Plastic Surgery, Kobe University, Chuo, Kobe, Hyogo, Japan; ${ }^{3}$ South Australian Institute of Ophthalmology and Discipline of Ophthalmology and Visual Sciences, University of Adelaide, South Australia, Australia; ${ }^{4}$ Division of Oculoplastic and Orbital Surgery, Department of Ophthalmology, Tel-Aviv Medical Center, Tel-Aviv University, Tel-Aviv, Israel
Correspondence: Hirohiko Kakizaki Department of Ophthalmology, Aichi Medical University, Nagakute, Aichi 480-II95, Japan

$\mathrm{Tel}+8|56| 6233 \mid \mathrm{I}$

Fax +8I 56I 637255

Email cosme@dI.dion.ne.jp
Purpose: To assess the effectiveness of the upper eyelid double motion sign in Graves' orbitopathy (GO) in detecting thyroid dysfunction or a positive level of thyroid-related autoantibodies.

Methods: GO was defined when more than two GO-related eyelid symptoms, including the double motion sign, existed with at least one positive thyroid-related blood test. Blood tests were performed in patients with more than two GO-related eyelid symptoms. The double motion was defined when the upper eyelid stopped at least once during downward eye movement. Fifty patients without GO or other eyelid diseases were used as controls.

Results: There were 353 patients who showed more than two GO-related eyelid symptoms including the upper eyelid double motion sign. Of these, 300 patients were diagnosed with GO $(300 / 353,85.0 \%)$. The double motion sign was demonstrated in 267 patients $(75.6 \%)$. A pause in double motion was typically seen around the anterosuperior direction of gaze. Double motion was not seen in any of the control eyelids. Although only $7.0 \%$ were hyperthyroid and $8.6 \%$ were hypothyroid, thyroid related autoantibodies were shown in $73.9 \%$ of patients. When the double motion sign was removed from the diagnostic criteria of GO, 263 patients had more than two thyroid-related eyelid symptoms, including 223 patients diagnosed as GO (25.7\% reduction), although the rate of a correct diagnosis was almost the same (84.8\%).

Conclusions: The double motion sign of the upper eyelids is frequently demonstrated in GO patients. This previously unreported sign can help in detecting thyroid dysfunction states with positive levels of autoantibodies.

Keywords: GO, self-antibody

\section{Introduction}

Graves' orbitopathy (GO) may precede, appear simultaneously to, or follow the actual symptoms of Graves' disease. ${ }^{1}$ In $10 \%$ of patients in whom orbitopathy appears first ("euthyroid Graves"), recognition of subtle signs and symptoms related to GO can sometimes be a useful tool in early diagnosis and effective treatment of Graves' disease. ${ }^{1,2}$

Almost all patients with thyroid dysfunctions are thought to have GO, including a mild symptom, ${ }^{1,3}$ although $25 \%-50 \%$ of patients with Graves' disease ${ }^{3}$ and 3\% of those with Hashimoto thyroiditis ${ }^{4}$ have symptoms of GO. While Graves' disease is easily diagnosed based on the typical signs and symptoms, ${ }^{1}$ the symptoms in hypothyroidism are much more subtle. ${ }^{4,5}$ It is not uncommon for the symptoms in 
hypothyroidism to initially be thought to be age related, or due to depression or dementia, ${ }^{6}$ which results in a delay in the correct diagnosis. ${ }^{4}$ Since hypothyroidism occurs in $10 \%-15 \%$ of adults older than 60 years of age, ${ }^{7,8}$ recognizing GO-related eyelid symptoms may help in an earlier detection.

GO-related symptoms may indicate the existence of thyroid dysfunction or thyroid-related self-antibodies. The typical eyelid symptoms in GO, such as Dalrymple's sign, Graefe's sign, Griffice's sign, and Enroth's sign, are usually easily detected, ${ }^{1}$ but can still be quite obscure in some cases.

In our practice, we noticed a unique eyelid sign in patients with GO, which we named "the upper eyelid double motion sign". This sign is noted during downward eye movement when Graefe's sign is analyzed. ${ }^{9}$ A pause in the double motion is seen around the anterosuperior direction of gaze. To the best of our knowledge, this double motion sign has not been reported previously.

In this study, we examined the effectiveness of the double motion sign as an additional GO-related eyelid sign for detection of thyroid dysfunction or a positive level of thyroid-related autoantibodies.

\section{Materials and methods}

This study was performed from September 2001 to December 2002 at a general eye clinic, not at a particular oculoplastic or a GO clinic.

In our study, we defined GO when more than two of the GO-related eyelid symptoms (Dalrymple's sign [upper eyelid retraction], Graefe's sign, Griffice's sign [lower eyelid lag], and Enroth's sign [eyelid swelling or fullness]) existed, including the double motion of the upper eyelids (defined as at least one pause during downward movement of the upper eyelid), ${ }^{1}$ in addition to at least one positive result from the following thyroid-related blood tests: thyroid-stimulating hormone (TSH) (normal range: 0.35-4.94 $\mu \mathrm{U} / \mathrm{mL}$ ), free T4 (normal range: $0.70-1.48 \mathrm{ng} / \mathrm{dL}$ ), free T3 (normal range: $1.71-3.71 \mathrm{pg} / \mathrm{mL}$ ), thyroglobulin (TG) (normal range: $30 \mathrm{ng} / \mathrm{mL}$ ), anti-TG antibody (anti-TGAb) (normal range: less than $0.3 \mathrm{IU} / \mathrm{mL}$ ), thyroid receptor antibody (TRAb) (normal range: $-15 \%-15 \%$ ), thyroid-stimulating antibody (TSAb) (normal range: less than 180\%), and antithyroid-peroxydase antibody (anti-TPOAb) (normal range: less than $0.1 \mathrm{IU} / \mathrm{mL}$ ). These thyroid-related blood tests were performed in patients with more than two GO-related eyelid symptoms, including the upper eyelid double motion sign.
Fifty volunteers (100 eyelids) without GO-related eyelid symptoms or other eyelid diseases were used as a control group. No thyroid-related blood tests were taken from these volunteers as they had no signs of GO or symptoms of thyroid dysfunction.

Consent to participate in the study was obtained from all patients and volunteers in the control group, after being informed of the purpose and outline of this study.

\section{Results}

A total of 353 patients (702 eyelids) had more than two GO-related eyelid symptoms including the upper eyelid double motion sign. Enroth's sign was demonstrated in 335 out of 353 patients (669 eyelids: 94.9\%), Griffice's sign in 210 patients (408 eyelids: 59.4\%), Dalrymple's sign in 102 patients (199 eyelids: 28.9\%), and Graefe's sign in 13 patients (23 eyelids: $3.7 \%$ ).

A total of 267 out of 353 patients (534 eyelids: $75.6 \%$ ) demonstrated the double motion sign (Figures 1A and 1B).

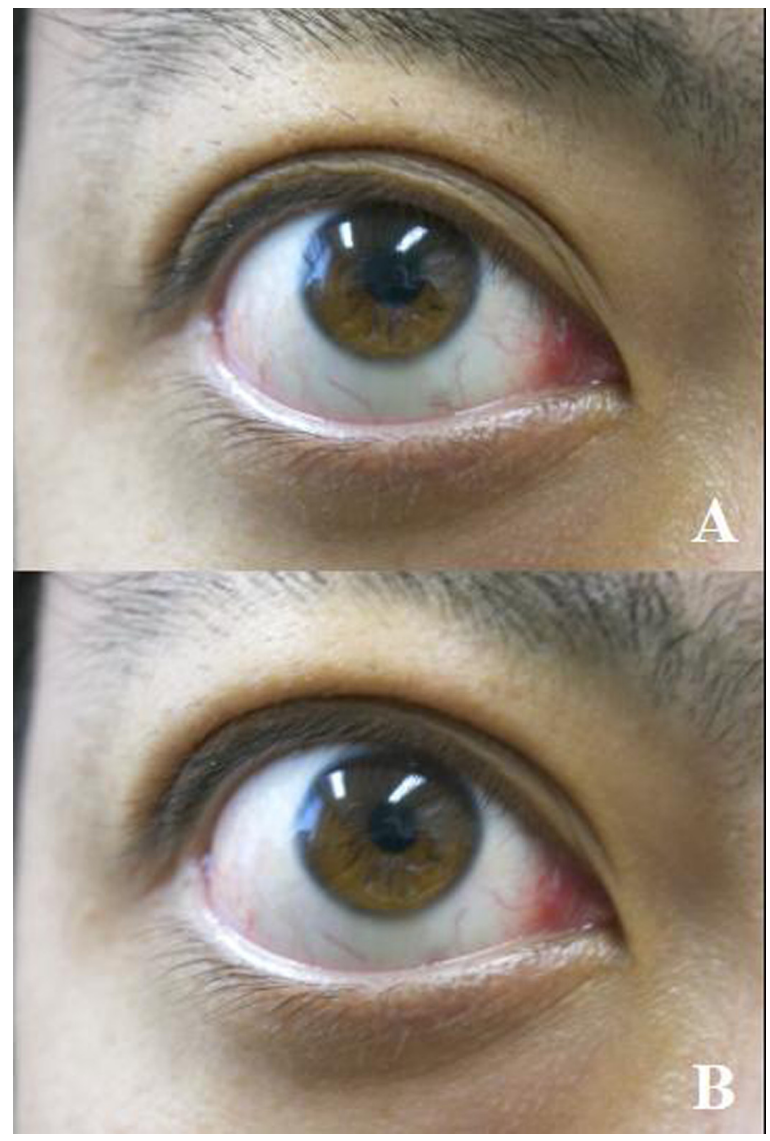

Figure I Double motion sign of the upper eyelid. A) Upper eyelid partially covers the upper part of the cornea on a slight upward gaze. B) The upper eyelid is still in the same position, but the eye moves downward, exposing the sclera between the upper eyelid and the cornea. 
Of these patients, 37 (13.9\%) were not diagnosed with GO (Table 1). The double motion was seen around the anterosuperior direction of gaze during downward eye movement. Although the Graefe's sign was shown in 13 patients (22 eyelids), multiple irregular pauses were demonstrated on downgaze. All eyelids used as control did not demonstrate the double motion sign.

A total of 300 patients out of 353 (85.0\%: total 596 eyelids; 107 males, 213 eyelids; 193 females, 383 eyelids) were diagnosed with GO, from which 253 patients (84.3\%) were euthyroid, 21 patients (7\%) were hyperthyroid, and 26 patients $(8.6 \%)$ were hypothyroid. The other blood tests were as follows: TSH (28 patients out of 353: 7.93\%), TG (105 patients, 29.7\%), anti-TGAb (59 patients, $16.7 \%$ ), TRAb (42 patients, 11.9\%), TSAb (209 patients, 59.2\%), and anti-TPOAb (168 patients, $47.6 \%$ ). Emergence of autoantibodies was $73.7 \%$ (260 out of 353 patients).

The sensitivity of the double motion of the upper eyelids in detecting GO was $76.7 \%$ and the specificity was $30.2 \%$.

When the double motion sign is not included in the diagnosis of GO, only 263 patients showed more than two GO-related eyelid symptoms, and 223 patients were diagnosed with GO $(25.7 \%$ reduction in the number of the GO patients), although the rate of a correct diagnosis was almost the same $(84.8 \%)$.

\section{Discussion}

The upper eyelid double motion sign was demonstrated in around $75 \%$ of patients. In addition, many patients demonstrated Enroth's (94.9\%) and Griffith (59.4\%) signs. Therefore, the double motion of upper eyelids sign, as well as the Enroth's and/or Griffith's signs are useful screening tools for ruling out thyroid dysfunction and the existence of thyroid-related autoantibodies. A total of 37 patients with negative blood tests also showed the double motion sign, and this can possibly be explained by the concept of "euthyroid Graves' disease".,1,2

The sensitivity of the double motion of the upper eyelids sign in detecting GO was $76.7 \%$, and the specificity was

Table I The relationship between the double motion sign of the upper eyelids and thyroid eye disease

\begin{tabular}{lllll}
\hline & & \multicolumn{2}{l}{ Graves' orbitopathy } & Total \\
\cline { 3 - 4 } & & Positive & Negative & \\
\hline Double motion & Positive & 230 & 37 & 267 \\
& Negative & $70^{\mathrm{a}}$ & 16 & 86 \\
& Total & 300 & 53 & 353 \\
\hline
\end{tabular}

Note: 'Thirteen patients demonstrated the Graefe's sign.
$30.2 \%$. Hence, when the double motion sign is positive, $76.7 \%$ of patients (a relatively high percentage), were diagnosed with GO. However, since the specificity is not as high, even when the double motion sign is negative, GO cannot be excluded. Although this may be a drawback, the existence of other GO-related symptoms may compensate for the low specificity.

In patients with a positive Graefe's sign, which may represent a more severe degree of inflammation or fibrosis of the levator palpebrae superioris muscle (LPSM) compared with the simple upper eyelid double motion sign, there were more frequent pauses or multiple motions except for the slightly upward site of the primary eye position. Based on this, the double motion of the upper eyelids may actually be a mild or subtle presentation of Graefe's sign.

The pause in the double motion, which is located around the anterosuperior direction of gaze, may be induced by a transient weak traction between the superior rectus muscle (SRM) and the LPSM. As communicating fibers connect the SRM to the LPSM, ${ }^{10}$ the upper eyelid moves synchronously with the SRM. ${ }^{10}$ However, when the LPSM changes its direction from forward to downward, ${ }^{10}$ the tension of the communicating fibers may be loosened at the point. Enhanced by the limited extension of the LPSM in GO, downward movement of the upper eyelids may be in delay to the globe movement around the position where the SRM traction is weaker.

Most patients in the present study $(84.3 \%)$ were euthyroid and only a minority ( $7 \%$ and $8.6 \%)$ were hyperthyroid and hypothyroid, respectively. Most of them were also diagnosed with GO without any general symptoms. In the general population, it is well documented that most patients with GO are hyperthyroid. ${ }^{11-13}$ A study from the Mayo Clinic $^{13}$ reported that $90 \%$ were hyperthyroid, $0.8 \%$ were hypothyropid, 5.8\% were euthyroid, and 3.3\% were diagnosed with Hashimoto thyroiditis. Since our study analyzed patients from a general eye clinic, and not from a particular oculoplastic or a GO clinic, this may explain the differences in the percentages.

Dalrymple's sign (upper eyelid retraction) was shown in only $28.9 \%$ of patients in the present study. In general, Dalrymple's sign is most frequently demonstrated as a GO-related eyelid symptom. ${ }^{14}$ One of the causes of upper eyelid retraction is increased sympathetic activity, ${ }^{14}$ which may be due to the effect of a high level of catecholamines in hyperthyroidism. ${ }^{11,15}$ As hyperthyroidism was noted in only $7 \%$ of patients in the current study, it may explain the low frequency of upper eyelid retraction. 
When the double motion sign was not included in diagnostic criteria for GO, only 223 patients were diagnosed with GO (25.7\% reduction in the number of the GO patients). This actually means that patients with Graves' disease may have been left without any treatment. Diagnosing Graves' disease is especially important in older patients since the symptoms of the disease in this age group are not always clear.

In conclusion, the double motion sign, which may represent a possible mild form of Graefe's sign, was frequently shown in GO patients but not in the control group. The sign is typically seen on a slightly upward site of the primary eye position during downward eye movement. The sign can help in detecting patients with thyroid dysfunction states and with positive levels of autoantibodies.

\section{Disclosure}

No authors have any financial/conflicting interests to disclose.

\section{References}

1. Wiersinga WM, Kahaly GJ, editors. Graves' Orbitopathy: A Multidisciplinary Approach. Basel, Switzerland: Karger; 2007.

2. Bartalena L, Pinchera A, Marcocci C. Management of Graves' ophthalmopahty: reality and perspectives. Endcr Rev. 2000;21:168-199.

3. Bahn RS, Heufelder AE. Pathogenesis of Graves' ophthalmopathy. N Engl J Med. 1993;329:1468-1475.
4. Perros P, Dickinson AJ, Kendall-Taylor P. Clinical presentation and natural history of Graves' ophthalmopathy. In: Bahn RS, editor. Thyroid Eye Disease. Boston, MA: Kluwer Academic Publisher; 2001: 119-136.

5. DeSanto LW. The total rehabilitation of Graves' ophthalmopahty. Laryngoscope. 1980;90:1652-1678.

6. Devdhar M, Ousman YH, Burman KD. Hypothyroidism. Endocrinol Metab Clin North Am. 2007;36:595-615.

7. Chiu AC, Sherman SI. Clinical manifestations and differential diagnosis of hypothyroidism. In: Falk SA, editor. Thyroid Disease: Endocrinology, Surgery, Nuclear Medicine, and Radiotherapy. 2nd edition. Philadelphia, PA: Lippincott-Raven; 1997: 379-392.

8. Kim G, Davies TF. Hypothyroidism. In: Besser GM, Thorner MO, editors. Comprehensive Clinical Endocrinology. 3rd edition. London, UK: Mosby; 2002:139-150.

9. Duke-Elder S, MacFaul PA. The ocular adnexa. In: System of Ophthalmology. London, UK: Henry Kimpton; 1974: 942-943.

10. Ettl A, Priglinger S, Kramer J, Koornneef L. Functional anatomy of the levator palpebrae superioris muscle and its connective tissue system. Br J Ophthalmol. 1996;80:702-707.

11. Gay AJ, Wolkstein MA. Topical guanethidine therapy for endocrine lid retraction. Arch Ophthalmol. 1966;76:364-367.

12. Ohno M, Hamada N, Yamakawa J, et al. Myasthenia Gravis associated with Graves' disease in Japan. Jpn J Med. 1987;26:2-6.

13. Bartley GB, Fatourechi V, Kadrmas EF, et al. Clinical features of Graves' ophthalmopathy in an incidence cohort. Am J Ophthalmol. 1996;121:284-289.

14. Kendler DL, Lippa J, Rootman J. The initial clinical characteristics of Graves' orbitpathy vary with age and sex. Arch Ophthalmol. 1993;111: 197-201.

15. Osanai H, Ohtsuka K, Nakamura Y, et al. Clinical Features of Graves' ophthalmopathy [Japanese]. J Eye. 1998;15:1043-1047.
Clinical Ophthalmology

\section{Publish your work in this journal}

Clinical Ophthalmology is an international, peer-reviewed journal covering all subspecialties within ophthalmology. Key topics include: Optometry; Visual science; Pharmacology and drug therapy in eye diseases; Basic Sciences; Primary and Secondary eye care; Patient Safety and Quality of Care Improvements. This journal is indexed on Submit your manuscript here: http://www.dovepress.com/clinical-ophthalmology-journal

\section{Dovepress}

PubMed Central and CAS, and is the official journal of The Society of Clinical Ophthalmology (SCO). The manuscript management system is completely online and includes a very quick and fair peer-review system, which is all easy to use. Visit http://www.dovepress.com/ testimonials.php to read real quotes from published authors. 